\author{
Milena Anđelić \\ Université de Belgrade - Faculté de Philologie \\ milenandelic@hotmail.com
}

\title{
QUELQUES COMPOSÉS PHYTONYMES
}

\begin{abstract}
Résumé : Ce travail porte sur les phytonymes français résultant du processus de composition et de la structure partie du corps + préposition de + nom d'animal. Il s'agit de composés agglomératifs exocentriques dont la structure syntaxique est gardée telle quelle lors de la substantivisation et dont le sens global ne dépend aucunement du sens des éléments morphologiques. Les noms de plantes étudiés ont pour motivation la ressemblance de certaines parties de la plante - référent avec les parties $d u$ corps de certains animaux. De même, nous pourrons observer que la taxinomie scientifique, en latin et en grec, est très proche des appellations usuelles françaises.
\end{abstract}

Mots-clés : phytonymie, composition, composés agglomératifs, composés exocentriques, métaphore, polysémie, analogie.

\begin{abstract}
This paper focuses on French phytonyms resulting from the composition process and having the structure 'body part term + preposition of + animal name'. These are exocentric agglomerative compounds whose syntactic structure is kept as it is during substantivization and whose overall meaning does not depend on the meaning of the morphological elements. The names of plants under analysis are motivated by the resemblance of certain parts of the plant to the body parts of some animals. Similarly, we can observe that the scientific taxonomy, in Latin and in Greek, is very close to the usual French appellations.
\end{abstract}

Keywords: phytonymia, composition, agglomerative compounds, exocentric compounds, metaphor, polysemy, analogy.

\section{Introduction}

Au prime abord, il peut sembler très facile de deviner de quelle plante parle une personne ou un auteur de roman lorsqu'ils mentionnent un pied-d'alouette, une patte-de-lion ou encore un cour-de-pigeon. En consultant un dictionnaire de langue, on obtient une définition qui ne suggère pas grand-chose à un amateur, la définition comportant en général un synonyme usuel ou normalisé tout aussi déroutant. Si on veut aller plus loin et ouvrir une encyclopédie, un ouvrage spécialisé, on peut voir même une image de la plante en question. Cela nous éclaire un petit peu, mais le problème se pose lorsque le dictionnaire et l'ouvrage spécialisé offrent plusieurs définitions - renvoient donc à plusieurs plantes... On se rend compte 
que la taxonomie botanique y est pour quelque chose : avec un nom de genre et d'espèce en latin ou en grec, un botaniste sait exactement de quel végétal il s'agit. En ce qui concerne les noms usuels (vernaculaires, populaires) des plantes, nous nous imaginons bien que dans un village ou une région, les personnes attribuent un signifiant, parfois deux, à un seul référent. Dans ces conditions d'utilisation restreinte, la confusion est impossible. Pourtant, rien ne nous garantit que les habitants d'une autre région aient choisi le même nom pour le même référent.

Dans ce travail, nous allons nous pencher sur les phytonymes usuels en français composés de plusieurs éléments : une partie de corps et un nom d'animal reliés par la préposition de. D'abord, nous allons étudier leur forme du point de vue morphologique et syntaxique, puis éclairer quelques points sémantiques.

Ensuite, il faut mentionner que les composés de la forme que nous avons choisie (1) n'appartiennent pas tous au champ lexical des végétaux, (2) qu'ils peuvent être polysémiques, les sens renvoyant à différents végétaux, (3) et qu'ils peuvent être phytonymes par certains de leurs sens seulement. Le sémantisme de chacun des éléments morphologiques est important, de même que le fait qu'il existe des phytonymes de structure similaire.

Dans le dernier chapitre, nous allons essayer de découvrir quelle partie de la plante a motivé la forme du composé et commenter la similitude des noms scientifiques des plantes avec les noms usuels français.

Pour trouver la définition de chaque composé, nous avons utilisé seulement les dictionnaires monolingues Dictionnaire Larousse en ligne (DL) et Trésor de la langue française informatisé (TLFi).

Ce travail vise à éclairer quelques points dans le domaine de la sémantique et ne prétend aucunement à une précision absolue dans le domaine de la botanique.

\section{La forme et le sens du composé}

Les phytonymes du type partie du corps + préposition de + nom d'animal sont formés, par le processus de composition, de trois morphèmes libres - deux noms reliés par une préposition. L'orthographe de ces noms n'est en général pas fixe et ils peuvent s'écrire avec ou sans trait d'union'. Seul le premier élément reçoit la marque du pluriel. Les substantifs composés sont du même genre que leur premier élément (un pied-d'alouette, une dent-de-lion), mais parfois l'autre genre est aussi possible (un dent-de-lion).

La structure syntaxique qu'on peut observer dans ces composés est SN + SP, le syntagme prépositionnel étant complément de nom du syntagme nominal. Il s'agit donc de composés agglomératifs, où la structure entière est prise telle quelle dans le mécanisme de substantivisation.

${ }^{1}$ Nous allons opter pour l'orthographe avec le trait d'union, pour mieux expliciter qu'il s'agit de composés. 
En ce qui concerne les spécificités sémantiques, nous allons mentionner la différence entre la sémantique intérieure et la sémantique extérieure ou globale. La sémantique intérieure découle directement de la structure syntaxique décrite ci-dessus : le premier élément partie du corps est en relation génitive de possession avec le troisième élément animal, ce qui est rendu possible par l'utilisation de la préposition de dans le sens de « appartient à ». Du point de vue de la sémantique globale du composé, en revanche, nous remarquons qu'un nom de plante n'a aucun lien formel avec des parties du corps des animaux : le sémantisme des morphèmes ne joue aucun rôle dans le sémantisme du composé. Il s'agit donc de composés exocentriques. Effectivement, ces phytonymes sont désignés par les parties du corps des animaux par analogie, en raison de la ressemblance d'un ou plusieurs aspects du premier et du troisième élément ${ }^{2}$.

\section{Composés similaires : structure et polysémie}

\subsection{Les composés non phytonymes}

Tous les composés du type partie du corps + préposition de + nom d'animal ne désignent pas des plantes. Certains d'entre eux sont des noms d'outils divers : pied-de-biche (désigne plusieurs sortes de leviers), pied-de-roi (règle pliante), queue-de-rat (lime ronde)... ; des objets ou motifs décoratifs : dent-de-loup (bordure dentelée réalisée à l'or), pied-de-biche (pied de meuble façonné dans le style Régence ou Louis XV)... ; des vêtements ou des tissus : pied de mouche (tissu de laine ou de coton), queue-de-morue (habit de cérémonie), etc.

\subsection{Les composés phytonymes polysémiques}

Étant donné que ces composés sont généralement d'origine populaire, certains d'entre eux sont très polysémiques. Les composés désignant une plante peuvent donc aussi désigner diverses entités, plantes ou non :

pas-d'âne : 1. Tussilage : composée vivace à grandes feuilles [...]2. Garde de l'épée couvrant toute la main. 3. Escalier dont les marches, parfois rampantes, ont une faible hauteur et un très large giron. 4. Instrument destiné à tenir ouverte la bouche des animaux qu'on examine ou qu'on opère.

D’autres composés peuvent être polysémiques dans le sens où ils peuvent désigner différentes plantes :

queue-de-renard: Nom usuel commun à l'amarante, au mélampyre, au vulpin, etc.

\footnotetext{
${ }^{2}$ Михаило Поповић, Лексичка структура франиуског језика, Београд, Завод за уџбенике,
} 2009, pp. 77-92. 
Dans des cas plus rares, une plante est désignée par plusieurs composés de structure partie du corps + préposition de + nom d'animal, par exemple :

foie-de-boeuf: Nom usuel de la fistuline [sorte de champignon].

langue-de-boeuf: 1 . Demi-pique ou poignard à lame plate et large, à pointe aiguë.

2. Nom usuel de la fistuline.

\subsection{Les variations sémantiques des trois morphèmes libres}

Il existe, par ailleurs, de nombreux phytonymes qui ont une structure légèrement différente, par exemple : barbe-de-Jupiter, cheveux-de-Vénus, oreille-deJudas où nous avons les éléments du type partie du corps + préposition $d e+$ nom propre. En comparant les composés qui nous intéressent avec ces exemples-ci, nous remarquons que les parties du corps humain, telles que jambe, main, cheveux, n'apparaissent pas dans les combinaisons avec l'élément nom d'animal.

Le phytonyme avec barbe comme premier élément, barbe-de-capucin, est intéressant parce qu' au premier abord nous ne sommes pas certains quelle est la structure juste de ce composé, ayant en vue la polysémie du nom capucin : 1. Religieux d'une branche de l'ordre mendiant exempt des Frères mineurs. 2. Singe cébidé d'Amérique centrale, vivant en troupes. 3. Nom communément donné au lièvre par les chasseurs. 4. Nom que les oiseleurs donnent à divers petits oiseaux du genre munie. 5. Pigeon ayant un capuchon de longues plumes qui interdit toute vision latérale.

Le problème est vite résolu, parce qu'un lièvre, un petit oiseau ou un pigeon n'ont certainement pas de «barbe »; de même, il est peu probable qu'un phytonyme populaire soit motivé par un nom de singe d'Amérique centrale. Le premier sens cité est le plus probable. Donc, la structure du phytonyme barbe-de-capucin n'appartient pas à celle que nous avons choisi d'analyser, puisqu'un capucin, dans ce contexte, n'est pas un animal.

En ce qui concerne la préposition de, l'élément reliant les deux substantifs, nous allons seulement mentionner que la préposition $\grave{a}$ s'utilise dans certains autres phytonymes, en gardant le même sens de possession que de: par exemple, la bourse à pasteur est à comprendre comme « bourse de pasteur », « bourse qui appartient au pasteur ».

\section{Variation sociolinguistique des phytonymes}

Le nom scientifique ou binomi(n)al, en latin ou en grec, est celui qui est utilisé internationalement par toute la communauté scientifique. Le nom usuel (vernaculaire, populaire) est l'appellation traditionnelle dans une langue vivante, alors que le nom vulgaire est en général un calque du nom scientifique, considéré comme plus précis que le nom usuel et utilisé par les connaisseurs des plantes. Le nom vulgaire devient normalisé lorsqu'il est accepté par la communauté 
scientifique d'un pays comme l'équivalent du nom scientifique dans la langue vivante en question ${ }^{3}$.

\section{Motivation des composés}

Dans cette partie, nous allons répartir les phytonymes de la forme partie $d u$ corps + prép. de + nom d'animal dans les tableaux suivant la composante partie $d u$ corps. Nous indiquerons aussi, si possible, les synonymes ${ }^{4}$ tirés de $D L$ et $T L F i$, un exemple de nom scientifique tiré des ouvrages de référence et la motivation possible du composé. Dans les remarques, nous allons commenter la similitude entre le nom scientifique et le composé lui-même à l'aide des dictionnaires latin-français et grec ancien-français.

En ce qui concerne les synonymes vulgaires et les noms scientifiques, il faut préciser que c'est le nom scientifique (latin ou grec) du genre de la plante qui est plus souvent calqué en français en tant que nom vulgaire que le nom de l'espè$c e$. Puisqu'un genre peut compter des centaines d'espèces, tous nos composés analysés sont nécessairement polysémiques du point de vue de la systématique végétale, à quelques exceptions près.

\begin{tabular}{|l|l|l|l|}
\hline barbe & $\begin{array}{l}\text { synonymes (vulgaires } \\
\text { ou normalisés) }\end{array}$ & ex. de nom scientifique & motivation \\
\hline barbe-de-bouc & spirée & Spirea salicifolia & $\begin{array}{l}\text { disposition des fleurs } \\
\text { de l'inflorescence }\end{array}$ \\
\cline { 2 - 4 } & salsifis & $\begin{array}{l}\text { Tragopogon } \\
\text { porrifolius }\end{array}$ & forme de la fleur \\
\cline { 2 - 4 } & scorsonière & Scorzonera hispanica & $\begin{array}{l}\text { forme de la fleur ou } \\
\text { disposition des tiges et } \\
\text { des feuilles }\end{array}$ \\
\cline { 2 - 5 } & clavaire (champignon) & Ramaria stricta & $\begin{array}{l}\text { forme du champignon } \\
\text { entier }\end{array}$ \\
\cline { 2 - 4 } & hydne (champignon) & Hydnum repandum & $?$ \\
\hline barbe-de-chèvre & panicaut & Eryngium alpinum & forme de la fleur \\
\cline { 2 - 4 } & spirée & Spirea salicifolia & id. supra \\
\cline { 2 - 4 } & hydne & Hydnum repandum & id. supra \\
\hline barbe-de-renard & astragale épineux & Astragalus alopecurus & forme de la fleur \\
\hline
\end{tabular}

Remarque : Barbe-de-bouc et barbe-de-chèvre sont synonymes lorsqu'ils désignent soit le salsifis, soit l'hydne. L'hydne lui-même a encore un synonyme : pied-de-mouton, dont la motivation est plus évidente, puisque ce champignon ressemble à un sabot de mouton

${ }^{3}$ Miroslav M. Grandtner \& Marc-Alexandre Beaulieu, « Quelques principes de normalisation des noms botaniques français : les arbres d'Amérique du Nord», Meta, 55 (3), 2010, pp. 558-568.

${ }^{4}$ Nous avons éliminé des tableaux tous les sens non phytonymes, par manque de place. Nous avons seulement gardé quelques myconymes - noms de champignons, qui sont, dans ce cas, clairement indiqués. 
plus qu'à une barbe. Barbe-de-renard : le nom scientifique de l'espèce (alopecurus) vient du grec ( $\dot{\alpha} \lambda \omega \dot{\omega} \varepsilon \xi, \dot{\alpha} \lambda \omega \dot{\pi} \varepsilon \kappa \kappa \varsigma$, renard).

\begin{tabular}{|l|l|l|l|}
\hline dent & $\begin{array}{l}\text { synonymes (vulgaires } \\
\text { ou normalisés }\end{array}$ & ex. de nom scientifique & motivation \\
\hline $\begin{array}{l}\text { dent-de-brebis } \\
\text { ou } \\
\text { dent-de-cheval }\end{array}$ & gesse (variété de maïs) & $\begin{array}{l}\text { forme et couleur de la } \\
\text { graine }\end{array}$ \\
\hline dent-de-chien & erythrone & $\begin{array}{l}\text { Erythronium } \\
\text { dens-canis }\end{array}$ & $\begin{array}{l}\text { forme et couleur du } \\
\text { bulbe }\end{array}$ \\
\hline dent-de-lion & astragale épineux & Leontodon automnale & forme des feuilles \\
\hline
\end{tabular}

Remarque : Dent-de-chien : le nom d'une des espèces en latin, dens-canis, est le même que le nom usuel français. Dent-de-lion : une des variétés porte le nom scientifique de Leontodon, le même que le composé français.

\begin{tabular}{|l|l|l|l|}
\hline coeur & $\begin{array}{l}\text { synonymes (vulgaires } \\
\text { ou normalisés) }\end{array}$ & ex. de nom scientifique & motivation \\
\hline $\begin{array}{l}\text { coeur-de-pi- } \\
\text { geon ou } \\
\text { cœur-de-bœuf }\end{array}$ & $\begin{array}{l}\text { (variété de cerise à } \\
\text { chair ferme) }\end{array}$ & Prunus avium & $\begin{array}{l}\text { forme, couleur et } \\
\text { consistance du fruit }\end{array}$ \\
\hline
\end{tabular}

\begin{tabular}{|l|l|l|l|}
\hline gueule & $\begin{array}{l}\text { synonymes (vulgaires } \\
\text { ou normalisés) }\end{array}$ & ex. de nom scientifique & motivation \\
\hline $\begin{array}{l}\text { gueule-de-loup } \\
\text { ou } \\
\text { gueule-de-lion } \\
\text { ou } \\
\text { mufle-de-veau }\end{array}$ & muflier & Antirrhinum maius & forme de la fleur \\
\hline
\end{tabular}

\begin{tabular}{|l|l|l|l|}
\hline langue & $\begin{array}{l}\text { synonymes (vulgaires } \\
\text { ou normalisés) }\end{array}$ & ex. de nom scientifique & motivation \\
\hline langue-de-boeuf & $\begin{array}{l}\text { fistuline (champ.) } \\
\text { foie-de-boeuf }\end{array}$ & Fistulina hepatica & $\begin{array}{l}\text { forme et couleur du } \\
\text { champignon }\end{array}$ \\
\hline langue-d'agneau & $\begin{array}{l}\text { plantain } \\
\text { corne de cerf }\end{array}$ & Plantago media & $\begin{array}{l}\text { forme ovale des } \\
\text { feuilles }\end{array}$ \\
\hline langue-de-cerf & $\begin{array}{l}\text { scolopendre } \\
\text { (fougère) }\end{array}$ & $\begin{array}{l}\text { Asplenium } \\
\text { scolopendrium }\end{array}$ & forme des feuilles \\
\hline langue-de-chien & cynoglosse & $\begin{array}{l}\text { Cynoglossum } \\
\text { borbonicum }\end{array}$ & $\begin{array}{l}\text { forme et consistance } \\
\text { des pétales }\end{array}$ \\
\hline $\begin{array}{l}\text { langue-de- } \\
\text { serpent }\end{array}$ & ophioglosse (fougère) & $\begin{array}{l}\text { Ophioglossum } \\
\text { vulgatum }\end{array}$ & $\begin{array}{l}\text { forme ondoyante de } \\
\text { l'épi }\end{array}$ \\
\hline
\end{tabular}

Remarques : Langue-de-chien et langue-de-serpent : les noms scientifiques viennent du

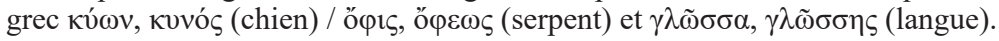


Milena Anđelić, Quelques composés phytonymes

\begin{tabular}{|l|l|l|l|}
\hline oreille & $\begin{array}{l}\text { synonymes (vulgaires } \\
\text { ou normalisés })\end{array}$ & ex. de nom scientifique & motivation \\
\hline $\begin{array}{l}\text { oreille-d'âne ou } \\
\text { oreille-de-vache }\end{array}$ & consoude & Symphytum officinale & forme de la fleur \\
\hline oreille-de-lièvre & $\begin{array}{l}\text { pézize (champ.) } \\
\text { =oreille-d'âne }\end{array}$ & $\begin{array}{l}\text { Otidea leporina } \\
\text { Otidea onotica }\end{array}$ & forme du champignon \\
\cline { 2 - 4 } & buplèvre en faux & Bupleurum falcatum & forme des feuilles \\
\hline $\begin{array}{l}\text { oreille-de-sou- } \\
\text { ris ou } \\
\text { oreille-de-rat }\end{array}$ & piloselle & Hieracium pilosella & $\begin{array}{l}\text { forme et taille des } \\
\text { pétales et des feuilles }\end{array}$ \\
\cline { 2 - 4 } oreille-d'ours & primevère auricule & Primula auricula & $\begin{array}{l}\text { forme et taille des } \\
\text { pétales et des feuilles }\end{array}$ \\
\hline
\end{tabular}

Remarques : Oreille-d'âne est synonyme d'oreille-de-lièvre lorsqu'il s'agit des champignons Otidea onotica (gr. ஸ̉ंíov, oreille + lat. onus, oneris, charge, fardeau) et Otidea

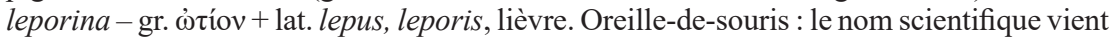

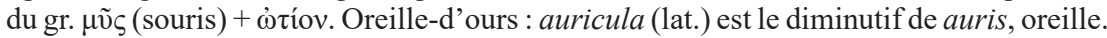

\begin{tabular}{|l|l|l|l|}
\hline pas / patte & $\begin{array}{l}\text { synonymes (vulgaires } \\
\text { ou normalisés) }\end{array}$ & ex. de nom scientifique & motivation \\
\hline pas-d'âne & tussilage & Tussilago farfara & forme des feuilles \\
\hline patte-d'araignée & nigelle des jardins & Nigella sativa & forme des feuilles \\
\hline patte-de-lapin & orpin velu & Sedum villosum & $\begin{array}{l}\text { présence de petits poils } \\
\text { sur la plante }\end{array}$ \\
\cline { 2 - 4 } & trèfle rouge & Trifolium pratense & disposition des feuilles \\
\hline patte-de-lion & alchémille & Alchemilla vulgaris & forme des feuilles \\
\hline patte-de-loup & lycope & Lycopus europaeus & forme des feuilles \\
\hline patte-d'oie & chénopode & $\begin{array}{l}\text { Dysphania } \\
\text { ambrosioides }\end{array}$ & forme des feuilles \\
\cline { 2 - 3 } & potentille & Potentilla anserina & \\
\hline
\end{tabular}

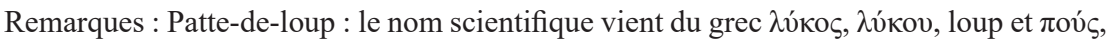

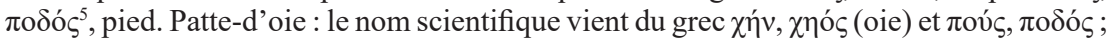
de même, le nom de l'espèce citée - anserina vient du latin anser, oie.

${ }^{5}$ Pour Lyco-pus, on a pris le nominatif grec, où nous ne voyons pas la forme complète de la base $(p u-)$; pour une autre plante (cf. pied-de-loup), Lyco-podos, on a pris la base entière (poD). Malgré la similitude des noms en français, ces phytonymes ne sont pas synonymes. 
ИСТРАЖИВАњА - RECHERCHES

\begin{tabular}{|l|l|l|l|}
\hline pied & $\begin{array}{l}\text { synonymes (vulgaires } \\
\text { ou normalisés) }\end{array}$ & ex. de nom scientifique & motivation \\
\hline pied-d'alouette & Delphinium elatum & forme des fleurs \\
\hline $\begin{array}{l}\text { pied-de- } \\
\text { corneille }\end{array}$ & (variété de plantain) & & $\begin{array}{l}\text { forme des grappes de } \\
\text { banane plantain (?) }\end{array}$ \\
\hline pied-de-chèvre & boucage & Pimpinella anisum & forme des feuilles \\
\hline $\begin{array}{l}\text { pied-de-poule } \\
\text { pied-de-coq }\end{array}$ & $\begin{array}{l}\text { (variété de renoncule } \\
\text { rampante) }\end{array}$ & Ranunculus repens & forme des feuilles \\
\cline { 2 - 3 } pied-de-lièvre & $\begin{array}{l}\text { trèfle des champs de chiendent) } \\
\text { =patte-de-lapin }\end{array}$ & Cynodon dactylon & \\
\hline pied-de-lion & edelweiss & Leontopodium alpinum & forme de la fleur \\
\hline pied-de-loup & lycopode d'Europe & Lycopodium clavatum & forme des pousses \\
\hline pied-d'oiseau & & Ornithopus perpusillus & forme des feuilles \\
\hline pied-de-veau & arum & Arum maculatum & $\begin{array}{l}\text { forme de l'axe floral } \\
\text { (?) }\end{array}$ \\
\hline
\end{tabular}

Remarques : Pied-de-lion et pied-d'oiseau : les noms scientifiques viennent du grec $\lambda \dot{\varepsilon} \omega v$,

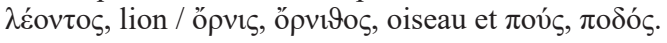

\begin{tabular}{|l|l|l|l|}
\hline queue & $\begin{array}{l}\text { synonymes (vulgaires } \\
\text { ou normalisés) }\end{array}$ & ex. de nom scientifique & motivation \\
\hline queue-de-renard & amarante & Amaranthus caudatus & forme de \\
\cline { 2 - 3 } & mélampyre & Melampyrum arvense & l'inflorescence \\
\cline { 2 - 3 } & vulpin & Alopecurus pratensis & \\
\hline
\end{tabular}

Remarques : Le nom scientifique du vulpin (lui-même de lat. vulpes, renard), Alopecurus, vient du grec ( $<\dot{\alpha} \lambda \omega \dot{\omega} \pi \varepsilon \xi$, $\dot{\alpha} \lambda \omega ́ \pi \varepsilon \kappa \varsigma_{\text {, }}$, renard). Le nom de l'espèce caudatus de l'amarante est issu du lat. cauda - queue.

\section{Conclusion}

Les parties du corps utilisées comme premier élément de la composition sont : la barbe, la dent, le cœur, la gueule, le mufle, le foie, la langue, la corne, l'oreille, le pas, la patte, le pied et la queue. Les parties du corps strictement humaines n'apparaissent pas.

Certains composés ayant comme éléments une partie du corps similaire et les noms d'animaux qui se ressemblent peuvent être synonymes, par exemple : pied-de-lièvre $=$ patte-de-lapin $;$ oreille-de-souris $=$ oreille-de-rat. Mais ceci n’est pas la règle : pied-de-lion : edelweiss / patte-de-lion : alchémille. Certains 
synonymes sont étonnants : cœur-de-pigeon = cœur-de-bœuf ; gueule-de-loup = gueule-de-lion $=$ mufle-de-veau.

Les noms d'animaux qui viennent en deuxième élément sont en général les animaux domestiques ou les animaux sauvages d'Europe, à l'exception du lion.

Ce que nous pouvons encore constater à partir des données que nous avons dans les définitions des phytonymes, c'est que, lorsqu'un phytonyme est polysémique, les plantes qu'il désigne sont généralement très différentes du point de vue scientifique, mais peuvent parfois être confondues par des amateurs. Les parties de la plante qui peuvent servir de motivation des composés sont les fleurs et les inflorescences, les pétales, les feuilles, les fruits, les tiges, l'aspect général, les signes particuliers (poils), rarement la racine. Quand un composé désigne différentes plantes, les parties de ces plantes servant de motivation peuvent aussi différer.

Le fait que beaucoup de ces composés ont les mêmes noms dans différentes langues européennes nous pousse à nous poser la même question que Wirth-Jaillard, à savoir : «s'il s'agit de formations parallèles [...] ou de calques d'une formation initiale $»^{6}$. En plus, la similitude entre certains composés usuels et leurs noms scientifiques montre explicitement qu'il s'agit de calques. Pourtant, il nous est impossible de conclure définitivement si c'est le nom usuel qui a motivé le calque scientifique ou si la situation est inverse.

\section{BIBLIOGRAPHIE}

Bailly, Anatole, Le Grand Bailly. Dictionnaire grec français, Hachette, Paris, 2000.

Bryant, Geoff et al, The Ultimate Plantbook, Global Book Publishing, Willoughby, 2005.

Claisse, Renée, Foucault, Bruno de, Delelis-Dusollier, Annick, « Nommer les plantes et les formations végétales », L'Homme [en ligne], 153, 2000, pp. 173-182.

Forey, Pamela \& Fitzsimons, Cecilia, Arbres, Librairie Gründ, Paris, 1988.

Forey, Pamela \& Fitzsimons, Cecilia, Fleurs sauvages, Librairie Gründ, Paris, 1988.

Gaffiot, Félix, Le Grand Gaffiot. Dictionnaire latin-français, Hachette, Paris, 2005.

Grandtner, Miroslav M. \& Beaulieu, Marc-Alexandre, « Quelques principes de normalisation des noms botaniques français : les arbres d'Amérique du Nord », Meta, 55 (3), 2010, pp. 558-568.

${ }^{6}$ Aude Wirth-Jaillard, « Entre linguistique et botanique : l'étymologie des noms de plantes. L'exemple des noms de cactus. " in Actes du XXVII Congrès international de linguistique et de philologie romanes, éd. S. N. Dworkin, X. L. García Arias, J. Kramer, 2013, p.186. 
Hochard-Bihannic, Sandra, « Analyse lexico-sémantique de la phytonymie populaire hispano-américaine », Acta Botanica Gallica, 155(2), 2008, pp. 317-320.

Поповић, Михаило, Лексичка структура франиуског језика, Завод за уџбенике, Београд, 2009.

Vidal, Jules, « Systématique, Nomenclature et Phytonymie botanique populaire au Laos ", Journal d'agriculture tropicale et de botanique appliquée, vol. 10, n¹0-11, 1963, pp. 438-448.

Анчић, Војин В. (dir.), Велика књига о башти : планирање, обликовање, размножавање, ИКП „ЕВРО“, Београд, 2001.

Wirth-Jaillard, Aude, « Entre linguistique et botanique : l'étymologie des noms de plantes. L'exemple des noms de cactus. " in Actes du XXVII Congrès international de linguistique et de philologie romanes, éd. S. N. Dworkin, X. L. García Arias, J. Kramer, 2013, pp.181-190.

Dictionnaire historique de la langue française, Le Robert, Paris, 1992.

Dictionnaire Larousse français monolingue en ligne : www.larousse.fr.

TLF : Trésor de la langue française informatisé, CNRS édition, Paris, 2004.

Милена Анђелић

\author{
НЕКОЛИКО СЛОЖЕНИЦА ФИТОНИМА \\ (Резиме)
}

У овом раду реч је о француским фитонимима насталим процесом композиције и са структуром део тела + предлог de + име животиње. Ради се о агломеративним и егзоцентричним сложеницама чија је синтаксичка структура сачувана у процесу поименичавања, а глобално значење им не зависи од морфолошких елемената. Проучавана имена биљака мотивисана су углавном сличношћу изгледа дела биљке - референта с деловима тела неких животиња, а видећемо да у научној таксономији на латинском и грчком језику имамо веома сличне називе као што су и на француском.

Кључне речи: фитонимија, композиција, агломеративне сложенице, егзоцентричне сложенице, метафора, полисемија, аналогија.

Примљено 4. септембра 2019, прихваћено за објављивање 10. децембра 2020. године. 